\title{
Impact of the Inter-relay Handoff on the Relaying System Performance
}

\author{
H. Nourizadeh, S. Nourizadeh, R. Tafazolli \\ Centre for Communication Systems Research (CCSR) \\ University of Surrey, UK
}

\begin{abstract}
It is foreseen that the next generation of cellular network would integrate the relaying or multihop scheme. In a multihop cellular architecture, the users are not only able to communicate directly to the base station (BS) but can also use some relay stations to relay their data to the BS. In such architecture, it may happen that a relayed user handover to another relay station during its communication: this process is called the inter-relay handoff. The main objective of this paper is to study how frequent the inter-relay handoff occurs and its impact on the relaying system performance. For this, different algorithms to decide when a user should inter-relay handover are proposed and tested through a dynamic system level simulator. We compare the capacity gain for the different algorithm with the conventional cellular networks using the UMTS FDD mode. The result showed that with an appropriate inter-relay handoff scheme, the uplink capacity gain of $\mathbf{3 5 \%}$ is readily achievable.
\end{abstract}

Index Terms-Relaying, CDMA, Capacity, inter-relay handoff, Cellular Networks, Mesh Networks.

\section{INTRODUCTION}

In a conventional cellular network, a base station (BS) controls a number of mobile station (MS) within its own coverage area and all the terminals communicate directly with the BS. However with an ever-increasing demand for mobile communications, we need to investigate new access schemes as well as novel system architectures to improve the offered cell capacities without increasing the required radio frequency spectrum. One of the main impediments to successful data transmission is error due to fading and interference. In the current conventional cellular networks, all MSs, even those far away from their BS have to communicate directly with the access point, requiring high levels of radiated powers and causing considerable interference to the neighboring cells. One solution would be to reduce a cell size and thereby increase the number of base stations. This way majority of users will be closer to a base station and would create less interference to others cells. However, this solution is not very economical due to significant increase in network infrastructure costs. Another possible solution to improve the capacity of cellular network is to use the concept of relaying in a cellular network. In a cellular network with relaying capability (also commonly known as multihop cellular network, or relaying system), the users are able to send their data directly to the BS or use some relay stations (fixed or mobile) to relay their data to the BS. In such architecture, the link is broken down into shorter paths requiring less power and hence creates less interference to the neighboring cell.

With less interference, more users can be accepted in the system and the capacity is increased. In addition to capacity improvement and power saving, another important benefit is the coverage enhancement. Due to the varying characteristics of a wireless environment, there are usually blind spots in the coverage. With the use of relaying the blind spots can be eliminated effectively and hence the coverage of the system can be enhanced.

It is clear from the above discussions that multihop architecture is a promising approach to the capacity and coverage improvement of a cellular network [1]. In the last few years many work have been done to qualify and quantify the capacity and coverage enhancement with relaying. For this, two different types of relaying network architecture have been studied. One suggested to use other users terminal to relay the traffic (Mobile Relay Station, MRS) [2]-[4], while others proposed to use Fixed Relay Station [5], [6]. The two different concepts are illustrated in Fig.1. Due to their different characteristics, each type of relay station (fixed or mobile) brings several advantages and disadvantages to the cellular networks, which are discussed in [7]. One of the main challenges with MRS is the frequent inter-relay handoffs. Due to the MS's mobility, the propagation channel condition between the relayed MS and their RS change. If the quality of the channel between MS and RS is getting worse, the relayed users would probably need to handover to another RS with better channel quality. This process, particular to the relaying system, is called the inter-relay Handoffs (IRHO). If the IRHO happen frequently, the relaying system will not be practical and the system performance might not be improved. The IRHO is an important issue when relaying is done through Mobile RSs, since these latter are constantly moving conversely to the Fixed RS. Therefore it is important to look at the impact that the IRHO can have on the system performance with relaying. In the literature review on relaying system, there are no works that treated this issue. In this paper, we focus on how frequent the IRHO occurs in the relaying system with MRS but also FRS, and on how much the inter-relay handover affects the system capacity especially when relaying with Mobile RS?

First, the description of the cellular networks with relaying with MRS and FRS are described in section II. Then different algorithms for the IRHO are proposed and explained in section III. The simulation's results on the impact of the IRHO on the system performance are presented in section IV. And a 
conclusion is drawn in section $\mathrm{V}$.

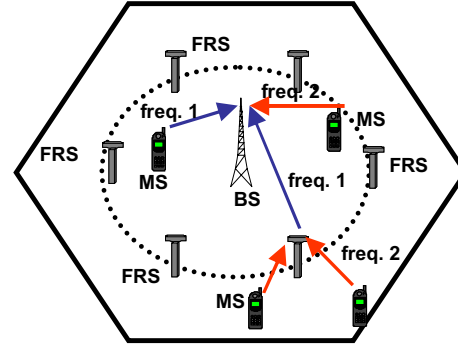

(a) with FRS

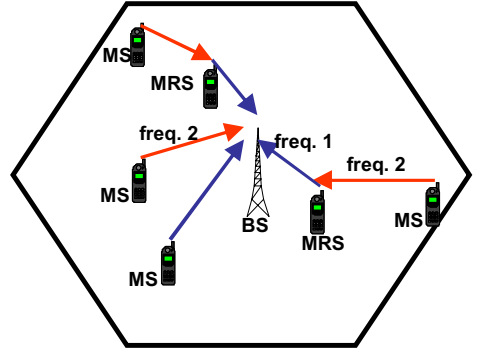

(b) with MRS
Fig.1. Cellular Networks with Relaying

\section{Cellular Networks with Relaying}

In order to study the impact of the IRHO on the relaying system performance, two relaying network architecture, one with MRS and one with FRS, are modeled. Their performances are compared with the conventional cellular networks. Therefore in this section, a detailed description of the cellular networks model with MRS and FRS are given for the uplink scenario.

\section{A. System description}

For both models (without and with relaying) all the assumptions are the same for fair comparisons. For example, a predetermined number of users are uniformly distributed over the whole system. After the handover mechanism, the users select the BS with the strongest pilot signal as the serving BS. Some users are considered active, which means they transmit information on the data channel while the rest are considered to be in an idle state.

In the non-relaying model, the active users transmit their data directly to their serving BS. The received Signal-toInterference Ratio (SIR) of each active user is calculated at the $\mathrm{BS}$ and the number of users in outage in the system is found. A user is considered in outage if its received SIR is below the SIR target. This process is repeated for several steps. One step (or snapshot) represents one location for all users. If the received SIR is below the SIR target for a certain number of steps, the user is assumed to be unsatisfied.

In the relaying model with Fixed or Mobile RS, the active users with bad channel quality relay their data to the BS through a relay station. References [Nou05a] showed the performance is optimal when the relaying is carried out for all the MS experiencing a SIR less than the SIR target in the direct link. Therefore only the MSs in outage will relay their data through a RS. The selection of the RS depends on whether the RS is fixed or mobile and it is explained in the next sub-sections. For simplicity, a maximum of two-hop communication (one hop for the MS-RS and one hop for the RS-BS communication) is considered in the relaying model.

In this paper, the MS transmit using the UMTS FDD air interface. Since a RS cannot transmit and receive at the same carrier frequency, two different carriers are needed in the relaying system. The non-relayed MSs transmit directly to the BS either with one carrier frequency (F1) or with a second carrier frequency (F2). While the RSs transmit to the BS with the frequency F1 and the relayed MS with the frequency F2 to the RS, see Fig. 1. In such a scenario, the same number of MSs should transmit in both carrier frequencies (F1 and F2). Since two carrier frequencies are used, in order to make fair comparison, the model is compared with the non-relaying system using also two carriers.

There is no interference between users transmitting in different carriers but only between the users transmitting in the same carrier frequency (F1 or F2).

In order to keep low the level of interference in the system, a perfect distributed power control is implemented. It consists of balancing all the received SIR around the SIR target. After the power control algorithm, the SIR of all the links MS-RS, RSBS and MS-BS are calculated. For a detailed calculation of the different SIR please refer to [8]. The relayed users are considered in outage if at least one of the two hops experiences a SIR below the SIR target. Thus the percentage of unsatisfied users in the relaying system can be calculated and compared to the non relaying case.

\section{B. Relaying model with Mobile RS}

In a cellular network with MRS, the potential candidates to relay the signals are the non-active users. Depending on the density of the non-active users, a relayed MS can select from a pool of users in idle state. However when a MS needs to be relayed, it must choose a suitable mobile relay station amongst the possible candidates. Not choosing the optimal relaying node can have great impact on the overall system capacity. Therefore different relaying MS selection schemes were proposed and evaluated in [8]. The results showed the best system performance is obtained when the non-active MS whom provide the strongest total SIR is selected as relay station. Total SIR means the sum of the SIR in the two hop links (MS-MRS and MRS-BS). In this model, we assume one MRS can relay only one MS at a time. Therefore, if two MSs want to relay with the same MS, the priority will be given to the one with the worse link quality (using path loss as criteria) with the BS. However, there are enough non-active MS are placed in the system that all the MS requiring relaying are able to find a relay station.

\section{Relaying model with Fixed RS}

In a cellular network with FRS, a number of stations are placed at specific locations in the cell so as to relay the signal of users in outage to the BS. Since there are only a few number of FRS in a cell, the relayed MS will have few choices to select a FRS. For that reason, the FRS selection is not an issue as important as with MRS, where there are many relay candidates. In our relaying system, the relayed MS will select among the two closest FRS, the one with whom it experiences the greatest SIR.

It may happen that several MS select the same FRS since there are few FRS deployed. However, we have realistically assumed the FRSs are able to relay several MS at a time. As a result, the FRS will send as much as data as the number of MS that it relays. This is taken into account in the model by increasing the data rate of each FRS in function of the number of relayed MS. As a consequence, the SIR target between the FRS and the BS will increase proportionally. Since a FRS relay the data of several MS, it is very important to have a 
good propagation channel between FRS and BS. In our relaying model, we assume the FRS located at strategic location where they experience a LOS with the BS and are equipped with directional antenna in order to reduce interference to the neighboring cells.

\section{INTER-RELAY HANDOVER SCHEME}

\section{A. IRHO schemes for MRS}

In a cellular network with MRS, all the are moving all the time. During the relaying communication, the link quality of the link MS-MRS or/and MRS-BS might drop under certain threshold. Consequently the relayed MS might need to change its MRS in order to achieve its traffic to the BS but also to minimize the overall interference in the system (Fig.2.). Therefore the decision on when a relayed MS should handover to another RS becomes critical (see Fig. 3). To answer theses questions, three algorithms were proposed and compared through the simulation's results.

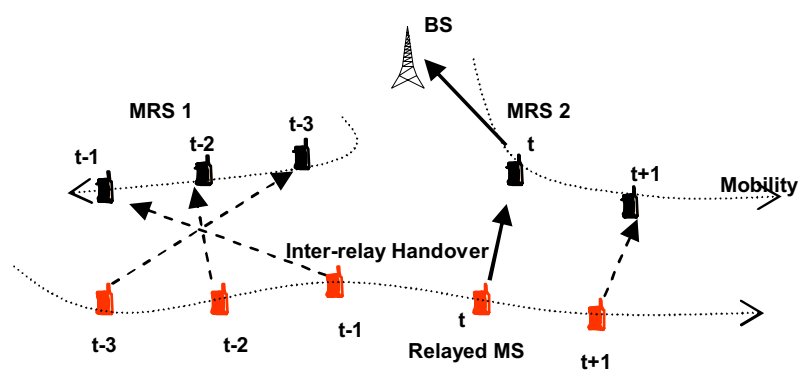

Fig. 2. Inter-relay HO due to mobility

\section{1) Inter-relay Handover Instantaneous (Inst-IRHO)}

The first proposed algorithm is called "InstantaneousIRHO". The relay station selection scheme proposed in [8] is based on the maximum total SIR. Whenever a MS requires relaying it will choose among the non-active MS the one with the maximum total SIR. Total SIR means the sum of the SIR in the two hop links (MS-MRS and MRS-BS). Therefore at each iteration, the relayed MS will inter-relay handoff if it finds another non-active MS with greater total SIR. For example in Fig. 3:

If Total SIR2 $>$ Total SIR1 $\rightarrow$ inter-relay handover from MRS1 to MRS2

\section{2) No Inter-relay Handover (No-IRHO)}

The second proposed algorithm is called "No-IRHO". In this scheme, when a MS selects a non active MS, it will keep the same MRS until it stops relaying. As a result IRHO will be avoided. However it is worth noting that the relayed MS will handover with another non-active MS only if its MRS moves to another cell.

\section{3) Inter-relay Handover based on requirement (SIR- IRHO)}

The third proposed algorithm is called "IRHO based on requirement (SIR-IRHO)". In this algorithm, IRHO is carried out only when the quality of the two-hop link drops below another link SIR with a certain margin. The relayed MS will inter-relay handover if there is another non-active MS with better link quality. In order to avoid frequent handover, the serving MRS will be changed only if the total SIR of another non-active MS is greater than the total SIR of the current MRS plus a SIR margin for IRHO:

If Total SIR2 $>$ Total SIR1 + SIRm_HO $\rightarrow$ inter-relay handover from MRS1 to MRS2

SIRm_HO is a margin value that decides on the inter-relay handover frequency.

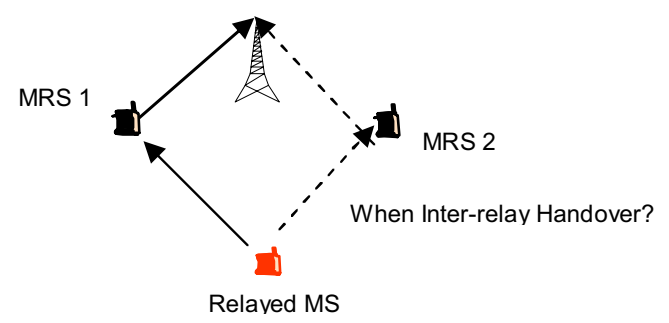

Fig. 3. Inter-relay Handover mechanism

\section{B. Relaying through Fixed Station}

In a relaying system with FRS, the inter-relay handover is affected only by the mobility of the relayed MS since the RS have a fixed location. Therefore the inter-relay handover has less impact on the system performance of the relaying system with FRS than with MRS. Since the FRS are located in a specific place where they can experience a very good propagation channel with the BS (e.g. LOS), the inter-relay handover will depend mainly on the link quality between the MS and FRS. In the relaying system with FRS, at each iteration the MS that requires relaying, will choose among the two closest FRS the one with whom it experiences the best SIR. Therefore it is unnecessary to implement different scheme (such as No-IRHO or SIR-IRHO) for inter-relay handover between FRS.

\section{IMPACT OF IRHO ON THE SYSTEM PERFORMANCE}

\section{A. Environment Model}

The system modeled includes 19 hexagonal cells of radius $2000 \mathrm{~m}$, with BS at the center of the cells employing omni directional antenna. The MS follow the mobility and the propagation model as proposed in [9] for the vehicular environment. An auto-correlated shadowing is considered between the MS and the BS. In the case of relaying with MRS, there are no appropriate path loss models that describe the channel between MSs since a mobile terminal is usually positioned around head. However, the Lee model, which takes into account the receivers antenna height, is chosen as model [10]. For the relaying case with FRS, the FRS are placed at 5m above the average rooftop and use the path loss model as in 
[9] for the MS-FRS link and the free space loss between FRSBS. The uplink scenario with the same fixed transmission rate for voice service is applied to all the active users and the relaying model is applied to all the 19 cells. The model is simulated at system level and the simulation has been run for 1000 correlated snapshots. The simulation result is based on the percentage of non-satisfied user. A user is considered nonsatisfied if its SIR is not above or equal to the SIR target - 0.5 $\mathrm{dB}$ for a certain period of time (the period chosen is 5 consecutive seconds from [9]). At the end of the simulation, the numbers of non-satisfied users were calculated without and with relaying (MRS and FRS) for the central cell and compared.

\section{B. Results}

\section{1) Impact of IRHO}

Each algorithm has been tested with dynamic system level simulator. For each algorithm, the probabilities of nonsatisfied users for different number of active users are plotted in Fig. 4. The three different algorithms are compared with the non-relaying system. For a probability of $2 \%$ of non-satisfied users, the non relaying system can support 92 users per cell.

First, it is important to note that the relaying system with any of the proposed scheme have a better performance than the non-relaying system. Among the three different proposed schemes, the best performance is obtained with the Inst-IRHO. By selecting the non active MS whom provide the strongest total SIR at any time, the interference in the system is minimized and the system performance is optimal. For $2 \%$ of non-satisfied users, the number of users accepted in the cell in the relaying system with Inst-IRHO is 136 , leading to $48 \%$ of capacity gain compare to the non-relaying system. However, this scheme does not consider the expenses due to frequent inter-relay handoffs.

It is seen that the second proposed scheme "No-IRHO" get the worse performance out of the three different algorithms. When a relayed MS selects a MRS, it will keep the same MRS until it does not need to relay. This is an efficient scheme to avoid IRHO but the relayed MS and its MRS are moving all the time, so their channel condition might not therefore be always the best. In a bad channel condition, the relayed MS and the MRS will increase their transmit power, which will increase the overall interference and the performance will not be optimal. Nevertheless, even with this scheme, the capacity is improved by $20 \%$ for $2 \%$ of non-satisfied users.

The third algorithm SIR-IRHO gets better performance than the No-IRHO scheme but less than the Inst-IRHO scheme. In this scheme, the relayed MS will inter-relay handoff only if the link quality with another MRS is much greater. Therefore, this scheme avoids frequent handovers such as in the InstIRHO scheme while providing a reasonable performance gain to the system. The capacity gain of the relaying system with SIR-IRHO depends on the SIRm_HO. The greater is the SIRm_HO, the less inter-relay handoff occurs. For a SIRm_HO equal to zero, the system performance is the same than the case with Inst-IRHO. For $2 \%$ of non satisfied users, the capacity gain with MRS is equal to $35 \%$ with a SIRm_HO of $14 \mathrm{~dB}$.

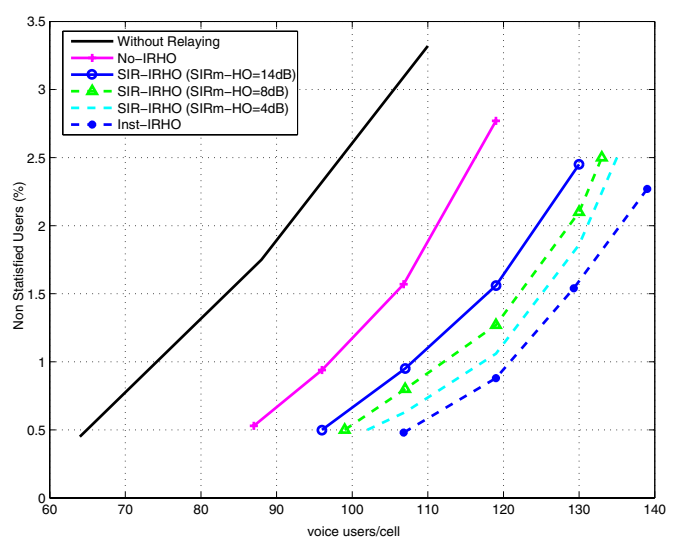

Fig. 4. Impact of Inter-relay Handover

2) Probability IRHO vs. MS speed

In Fig.4. the capacity gain provided by the different algorithm were shown without considering the number that IRHO happen. In this section, we compare at how frequent the inter-relay handover occurs for each algorithm. The probability of IRHO is the probability that a relayed MS change its RS during the relaying communication. It represents among the MS that were relaying, the number of relayed MS that handover to another RS. The probabilities of IRHO for different MS's speed with MRS and FRS are plotted in Fig. 5.

As expected, the Inst-IRHO is the scheme that causes the most inter-relay handover, which will considerably increase the signaling in the relaying system. The No-IRHO scheme will keep low the signaling at the expenses of a less gain in the system performance. A good compromise would be to employ the SIR-IRHO scheme. The result show that the probability of IRHO based on requirement is quite low in Fig. 5 while keeping a good capacity improvement in Fig.4. This algorithm is a good trade-off between the extra signaling and gain in the system performance.

Predictably, the probability of IRHO in the relaying system with FRS is quite low. One can see that the probability of IRHO with MRS based on requirement with a SIRm_HO of $14 \mathrm{~dB}$ is equal to the probability of IRHO with FRS. This shows that in this scheme, the relayed MS change MRS as often as in the relaying system with FRS. 


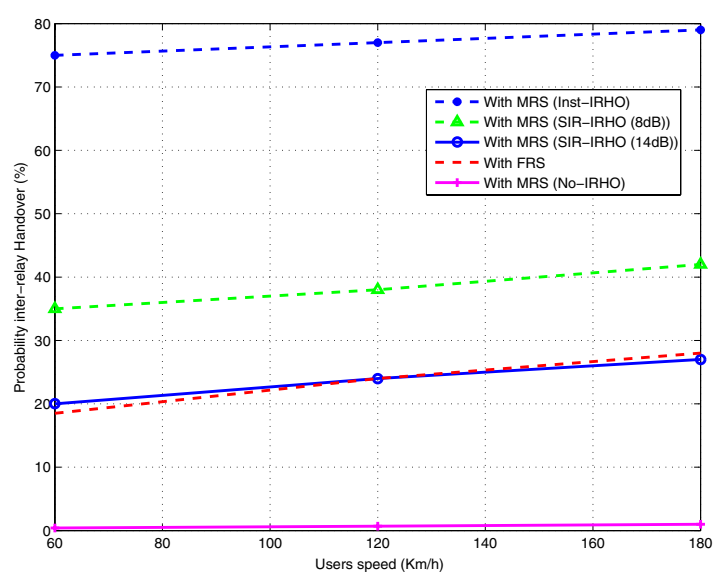

Fig. 5. Probability of inter-relay Handover vs. speed

\section{CONCLUSION}

In this paper, we studied the impact that the inter-relay handoff can have on the relaying system performance. The capacity of the conventional cellular network could be improved by 20 to $50 \%$ depending on the inter-relay handover scheme employed. The result showed that even when interrelay handover is not permitted (No-IRHO scheme); the capacity can still be improved by $20 \%$ with relaying. However, with an appropriate SIR window in the SIR-IRHO scheme, the relaying system with MRS can improve the capacity of the conventional cellular network as well as limiting frequent handoffs between the relay stations. The capacity of the cellular network with MRS is improved by $35 \%$ with the SIR-IRHO scheme.

\section{ACKNOWLEDGEMENT}

The authors would like to thank Vodafone UK to fund this research.

\section{REFERENCES}

[1] R. Pabst, B. Walke et al., "Relay-Based Deployment Concepts for Wireless and Mobile Broadband Radio", IEEE Communications Magazine, p.p. 80-89, New York, US, September 2004.

[2] ETSI.TR 101146 v3.0.0 "UMTS 30.06, Support for relaying and ODMA", December 1997.

[3] V. Sreng, Capacity Enhancement through Two-Hop Relaying in Cellular Radio Systems, Master's thesis, Carleton University, January 2002.

[4] J. Vidal et al., "Multihop networks for capacity and coverage enhancement in TDD/UTRAN", MedHocNet 2002, Sardegna, Italy, 5-7 September 2002.

[5] $\mathrm{H}$. $\mathrm{Hu}$ and $\mathrm{H}$. Yanikomeroglu "Performance Analsysis of Cellular Networks with Digital Fixed Relays" $10^{\text {th }}$ WWRF Meeting, NY, October 27-28 2003

[6] A. Molina, E. Tameh and A. Nix, "The Optimization of Fixed Relay Location to Enhance the performance of a 3G Microcellular Network" IST Mobile Communications Summit 2004, Lyon, France, 27-30 June 2004

[7] H. Nourizadeh, R. Tafazolli "Performance evaluation of Cellular Networks with Mobile and Fixed Relay Station", submitted to IEEE VTC fall, 26 Sept. 2006, Canada, Montreal

[8] H. Nourizadeh, R. Tafazolli "Capacity improvement of WCDMA cellular systems through different relaying strategies", MWCN 2005, Marrakech, Morocco, 19 September.
[9] ETSI (UMTS); selection procedures for the choice of radio transmission technology of the UMTS (UMTS 30.03 v3.2.0) 1998-04

[10] S.C Yang, CDMA RF Engineering, Artech House, Boston London, 1998. 